\title{
Advanced inertial position tracking of sensor particles in fluid flows of large industrial containers
}

\author{
Syed Shamsul Arefin ${ }^{1}$, Sebastian Felix Reinecke ${ }^{1}$, Uwe Hampel', ${ }^{2}$ \\ ${ }^{1}$ Institute of Fluid Dynamics, Helmholtz-Zentrum Dresden-Rossendorf e.V., Germany \\ ${ }^{2}$ AREVA Endowed Chair of Imaging Techniques in Energy and Process Engineering, \\ Technische Universität Dresden, Germany
}

s.arefin@hzdr.de

\begin{abstract}
In this paper, a concept for inertial position tracking of flow following sensor particles based on data fusion of inertial sensors is presented. The employed data fusion technique is quaternion based and uses an extended Kalman filter algorithm. A generalized sensor system kinematics has been developed to test the filter algorithm where three data conditions have been considered. Eventually, first simulation results are compared which shows the performance of the filter regarding sensor drift and noise are being discussed.
\end{abstract}

Keywords: Data fusion, Kalman filter, position tracking, sensor particle, flow follower.

\section{Introduction}

Increasing the efficiency of industrial processes is a long-running demand in terms of profitability and environmental sustainability. A specific problem is monitoring and control of conversion processes in large vessels. Such are to be found, e.g. in bio-chemistry, food production, minerals processing and biogas fermentation. Such often occur in large vessels and there typical problems are optimal homogenization of mass and heat by mixing, optimal suspension of solids, optimal volumetric mass transfer and reaction, efficient gas supply and others. Performing measurements in large vessel is often problematic or even not feasible. The concept of sensor particles has been developed in HZDR to investigate running processes in large vessels, such as biogas fermenters, bioreactors and activated sludge tanks. These sensor particles continuously collect spatially distributed hydroand thermodynamic process parameters using autonomous sensor technologies. Sensor particles are made up of robust capsules consisting of integrated electronics with sensor elements for basic physical parameters, microcontroller, memory, battery and a buoyancy control unit. Currently the sensor particle is equipped with an inertial measurement unit (IMU) consisting of integrated miniaturized sensors for acceleration, rotational rate and magnetic field, additionally the sensor particles also have sensors for ambient temperature, immersion depth as a function of hydrostatic pressure. The concept is also open to incorporate complementary miniaturized sensors, for example, sensors for $\mathrm{pH}$ and dissolved oxygen [1]. This work is focused on development of data fusion algorithms for position tracking of sensor particles using the available data from the integrated sensor elements.

Development of algorithms for integrating the gyroscope and accelerometer data, to estimate the attitude and position, gained increasing attention due to the various applications of autonomous systems. Studies related to positioning of robots [2], unmanned air vehicles [3], and underwater vehicles [5] have been performed. Also filtering methods using quaternion and Kalman filter have been applied in this context [6].

The uniqueness of this approach in application is, that the use of active position tracking or localization using radio waves or acoustics are not yet possible. GPS or acoustic techniques do not work well at typical process conditions, as scattering and attenuation of signal carriers in the process fluids limit the signal propagation too strongly. Moreover, the upper hand over these techniques is the availability of the a-priori knowledge, such as the use 
of magnetic markers or the structural knowledge of the container, which can be used to make precise position estimation. Theoretically, single and double integration of the gyro and accelerometer outputs may provide velocity and position information. But in practice the non-linearity and noise present in the sensors make the trajectory estimation effective for only a short period of time. The concept of data fusion, however, provides better control via noise models and gives a more precise estimation [3]. The detected position of a sensor particle in a time instant is in principle the fusion of data from gyroscope (rotational rate) and accelerometer provided an a-priori knowledge of the initial position. With the help of quaternion components to represent the orientation of the sensor particle and the magnetic field signal from the magnetometer, data fusion can be achieved to detect the position of the sensor particle by developing filter algorithms. Generally for the purpose of data fusion from multiple sensors, statistical filter algorithms are developed and the choice of the type of filter depends on the level of output efficiency required at application level. For this reason, the choice of a Kalman filter is sensible in our case. However, as we are dealing with an inertial sensor system, the equations of the system or the data mixing from the sensor is nonlinear. Thus it is not wise to use a linear Kalman filter. Instead, the idea is to use two other well-known versions of the Kalman filter, the EKF (Extended Kalman Filter) and UKF (Unscented Kalman Filter) [2]. The initial result presented in this paper is derived from EKF. Thus this kind of data fusion can give better control over the non-linearity and noise, which is always present in the system.

The goal of this study was to provide a system model that uses an extended Kalman filter algorithm to fuse sensor data using a precise noise model to estimate the position of sensor particles.

\section{EKF Model for Inertial Position Tracking}

In principle, the filter fuses data from a variety of sensor subsystems into a consistent, smooth estimate of the state of the sensor particle. The extended Kalman filter is nothing but a non-linear version of the typical Kalman filter and can provide a better estimate of the state vector by taking into account the nonlinearities present in the system. In the extended Kalman filter the system dynamics and the measurement function is linearized around the expected state and then the Kalman filter is applied [2,3]. For the implementation of EKF, a state space model of the sensor system has to be developed, which will be a non-linear function of the available outputs of the integrated sensors as shown in figure 1. This state space function is represented as the unobserved state vector, $\mathbf{x}_{\mathrm{k}}$, and is predicted over time using the acceleration, a, rotational rate, $\boldsymbol{\omega}$, from the gyroscope and quaternion $\mathbf{q}$. This can be achieved by propagating the state vector through a prediction function or state propagation function. The error covariance, $\mathbf{Q}_{\mathrm{k}}$, is predicted using the state propagating function and a state matrix, $\mathbf{A}$, can be derived with in the function for the purpose of propagation. The state vector is rotated using the rotation matrix, $\mathbf{R}_{\mathrm{b}}{ }^{\mathrm{n}}$, which is derived from the quaternion components. The observable measurements of each sensor are implemented into a non-linear measurement function and this is used to predict the measurement of the sensor and also an error covariance, $\mathbf{R}_{\mathrm{k}}$, of the measurement along with the measurement matrix, $\mathbf{H}$. By making use of the error covariance of the predicted state and the measurement, the Kalman gain is calculated. The predicted state vector and measurement vector along with the Kalman gain is then used to update or correct the state vector and the error covariance. This process of prediction and correction continues in every time step. This is illustrated in figure 1.

\section{State Space Model of the Sensor System}

The state space model is defined using the following nonlinear equations,

$$
\begin{gathered}
\mathbf{x}_{\mathrm{k}}=\mathbf{f}\left(\mathbf{x}_{\mathrm{k}-1}, \mathbf{u}_{\mathrm{k}-1}, \mathbf{w}_{\mathrm{k}-1}\right) \\
\mathbf{z}_{\mathrm{k}}=\mathbf{h}\left(\mathbf{x}_{\mathrm{k}}, \mathbf{v}_{\mathrm{k}}\right)
\end{gathered}
$$

where the unobserved system state vector, $\mathbf{x}_{\mathrm{k}}$, develops over time as a nonlinear function of the previous system state $\mathbf{x}_{k-1}$, control input, 
$\mathbf{u}_{\mathrm{k}-1}, \quad$ and process noise, $\mathbf{w}_{\mathrm{k}-1}$. The sensor measurements $\mathbf{z}_{k}$ are a nonlinear function of the system state in addition with measurement noise $\mathbf{v}_{\mathrm{k}}$. The process and measurement noises are assumed to be normally distributed white noise with zero mean and covariance $\mathbf{Q}_{\mathrm{k}}$ and $\mathbf{R}_{\mathrm{k}}$ respectively [2-4].

\section{Prediction and Measurement Stage}

To predict the position of the sensor particle, the variables that are taken into account are the quaternion components, represented by the rotational rates, $\boldsymbol{\omega}$, and the velocity, $\mathbf{v}$, of the sensor particle in body frame. The state of the sensor particle is represented by the following vector,

$$
\mathbf{x}_{k}=\left[\mathbf{P}^{\top}, \mathbf{q}^{*^{\top}}, \mathbf{v}^{\top}, \mathbf{b}_{\mathrm{a}}{ }^{\top}, \mathbf{b}_{\mathrm{g}}{ }^{\top}\right],
$$

where $\mathbf{P}$ is the 3-dimensional position vector in the body frame, $\mathbf{q}^{*}$ is the unit quaternion update representing the rotation of the body frame to the navigation frame, $\mathbf{v}$ is the translational velocity in body frame. Biases $\mathbf{b}_{a}$ and $\mathbf{b}_{\mathrm{g}}$ are $3 \times 1$ vectors representing the accelerometer and gyroscope drifting biases along each axis respectively. Data collected from the IMU are assumed to be in body frame, that is, the sensor particle's frame $[4,5]$.

The prediction stage of the EKF predicts forward in time, and the acceleration and gyroscope data is provided by the IMU which contains additional parameters such as the biases and the gravity compensation, that must be taken care off. These accelerometer and gyroscope data are then used within the state propagation function. The following equations shows the acceleration and gyroscope data along with the additional parameters. Acceleration in body frame is affected by gravity, $\mathbf{g}$, the drifting bias, $\mathbf{b}_{\mathrm{a}}$, and white noise $\mathrm{w}_{\mathrm{a}}$. The gyroscope data are affected by the drifting bias $b_{g}$ and the white noise $w_{g}[4,5]$.

$$
\begin{gathered}
\mathbf{a}=\left(\mathbf{a}_{\mathrm{b}}-\mathbf{b}_{\mathrm{a}}\right)+\mathbf{g}+\mathbf{w}_{\mathrm{a}} \\
\boldsymbol{\omega}=\left(\boldsymbol{w}_{\mathrm{b}}-\mathbf{b}_{\mathrm{g}}\right)+\mathbf{w}_{\mathrm{g}}
\end{gathered}
$$

These equations are now used for the state propagation function that is fed into the prediction stage of the extended Kalman filter.

$$
\left(\mathbf{x}_{k}\right)=\left(\begin{array}{c}
\mathbf{q}^{*} \\
\mathbf{P} \\
\mathbf{v} \\
\mathbf{b}_{\mathrm{a}} \\
\mathbf{b}_{\mathrm{g}}
\end{array}\right)=\left(\begin{array}{l}
\mathbf{R}_{\mathrm{b}}{ }^{\mathrm{n}} \boldsymbol{\omega} \\
\mathbf{v}_{\mathrm{b}}{ }^{\mathrm{n}} \\
\mathbf{R}_{\mathrm{b}}{ }^{\mathrm{n}} \mathbf{a} \\
0_{3 \times 1} \\
0_{3 \times 1}
\end{array}\right)
$$

The rotation matrix $\mathbf{R}_{b}{ }^{n}$ is formed using the unit quaternion $\mathbf{q}^{*}$. The unit quaternion is determined by the following method,

$$
\mathbf{q}^{*}=\mathbf{q}_{\mathrm{w}}+\mathbf{q}_{\mathrm{x}} \mathbf{i}+\mathbf{q}_{\mathrm{y}} \mathbf{j}+\mathbf{q}_{\mathrm{z}} \mathbf{k}
$$

where the quaternion norm is unity and the components are derived from the Euler angles [4],

$$
\begin{aligned}
& \mathbf{q}_{\mathrm{w}}=\cos (\varphi / 2) \cos (\theta / 2) \cos (\Psi / 2) \\
& \sin (\varphi / 2) \sin (\theta / 2) \sin (\psi / 2) \\
& \mathbf{q}_{\mathrm{x}}=\sin (\varphi / 2) \cos (\theta / 2) \cos (\Psi / 2) \\
& \cos (\varphi / 2) \sin (\theta / 2) \sin (\Psi / 2) \\
& \mathbf{q}_{\mathrm{y}}=\cos (\varphi / 2) \sin (\theta / 2) \cos (\Psi / 2) \\
& \sin (\varphi / 2) \cos (\theta / 2) \sin (\Psi / 2) \\
& \mathbf{q}_{\mathrm{z}}=\cos (\varphi / 2) \cos (\theta / 2) \sin (\Psi / 2) \\
& \sin (\varphi / 2) \sin (\theta / 2) \cos (\psi / 2)
\end{aligned}
$$

For a given set of gyroscope data, $\omega_{x}, \omega_{y}$, and $\omega_{z}$, the kinematic equation that represents the transformation from body frame to navigation frame is written as,

$$
\left(\begin{array}{l}
\mathbf{q}_{\mathrm{w}}{ }^{*} \\
\mathbf{q}_{\mathrm{x}}{ }^{*} \\
\mathbf{q}_{\mathrm{y}}{ }^{*} \\
\mathbf{q}_{\mathrm{z}}{ }^{*}
\end{array}\right)=\left(\begin{array}{cccc}
0 & -\omega_{\mathrm{x}} & -\omega_{\mathrm{y}} & -\omega_{\mathrm{z}} \\
\omega_{\mathrm{x}} & 0 & \omega_{\mathrm{z}} & -\omega_{\mathrm{y}} \\
\omega_{y} & -\omega_{\mathrm{z}} & 0 & \omega_{\mathrm{x}} \\
\omega_{\mathrm{z}} & \omega_{\mathrm{y}} & -\omega_{\mathrm{x}} & 0
\end{array}\right)\left(\begin{array}{l}
\mathbf{q}_{\mathrm{w}} \\
\mathbf{q}_{\mathrm{x}} \\
\mathbf{q}_{\mathrm{y}} \\
\mathbf{q}_{\mathrm{z}}
\end{array}\right)
$$


By making use of the quaternion components the rotation or the frame transformation matrix is determined by [5],

$\mathbf{R}_{\mathrm{b}}{ }^{\mathrm{n}}=$

$\left(\begin{array}{l}2\left(\mathbf{q}_{\mathrm{w}}{ }^{2}+\mathbf{q}_{\mathrm{x}}{ }^{2}\right)-1 ; 2\left(\mathbf{q}_{\mathrm{x}} \mathbf{q}_{\mathrm{y}}-\mathbf{q}_{\mathrm{w}} \mathbf{q}_{\mathrm{z}}\right) ; 2\left(\mathbf{q}_{\mathrm{x}} \mathbf{q}_{\mathrm{z}}+\mathbf{q}_{\mathrm{w}} \mathbf{q}_{\mathrm{y}}\right) \\ 2\left(\mathbf{q}_{\mathrm{x}} \mathbf{q}_{\mathrm{y}}+\mathbf{q}_{\mathrm{w}} \mathbf{q}_{\mathrm{z}}\right) ; 2\left(\mathbf{q}_{\mathrm{w}}{ }^{2}+\mathbf{q}_{\mathrm{y}}{ }^{2}\right)-1 ; 2\left(\mathbf{q}_{\mathrm{y}} \mathbf{q}_{\mathrm{z}}+\mathbf{q}_{\mathrm{w}} \mathbf{q}_{\mathrm{x}}\right) \\ 2\left(\mathbf{q}_{\mathrm{x}} \mathbf{q}_{\mathrm{z}}-\mathbf{q}_{\mathrm{w}} \mathbf{q}_{\mathrm{y}}\right) ; 2\left(\mathbf{q}_{\mathrm{y}} \mathbf{q}_{\mathrm{z}}+\mathbf{q}_{\mathrm{w}} \mathbf{q}_{\mathrm{x}}\right) ; 2\left(\mathbf{q}_{\mathrm{w}}{ }^{2}+\mathbf{q}_{\mathrm{z}}{ }^{2}\right)-1\end{array}\right)$

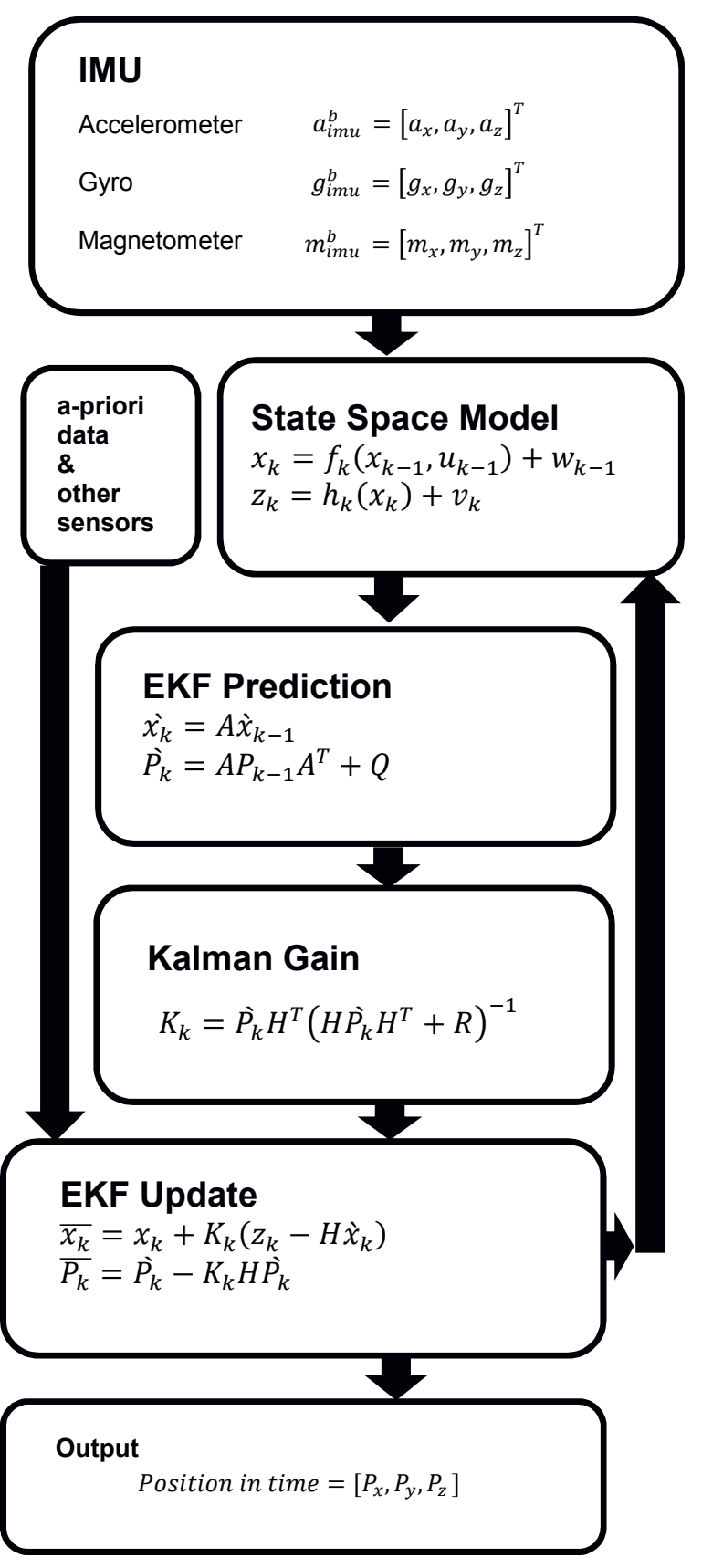

Fig. 1: Concept of filter algorithm for inertial position tracking of sensor particles using EKF.
The idea behind the development of the measurement function is the proper usage of secondary sensor data or a-priori knowledge to ensure that the estimated state vector in the process model can be corrected to move towards the expected state. Currently, the output data of the magnetometer are used for this purpose. The measurement model developed for our simulated system model is as following [2],

$$
\mathbf{z}_{\mathrm{k}}=\left[\mathbf{R}_{\mathrm{b}}{ }^{\mathrm{n}} \mathbf{m}_{\mathrm{b}} \mathbf{R}_{\mathrm{b}}{ }^{\mathrm{n}}\right]^{\top}
$$

where $\mathbf{m}_{\mathrm{b}}$ is the magnetometer output in body frame.

\section{Simulation Results and Discussion}

The filter algorithm is developed around a simple translatory motion derived to clearly understand the behavior of the movement pattern and the EKF itself. The accelerometer, gyroscope and the magnetometer data have been developed in MATLAB $尺$ as the input parameters of the EKF. Initial simulations were performed by only having a translatory motion in the y-direction without having any rotation in any of the axis. By using the simple strapdown method, the results will be very noisy or will have significant drift that would make the results unusable. In figure 2 , the red line is the result of simple strapdown integration and it can be seen that due to the presence of uncontrollable or unoptimizable noise, the result is drifting away from the expected one.

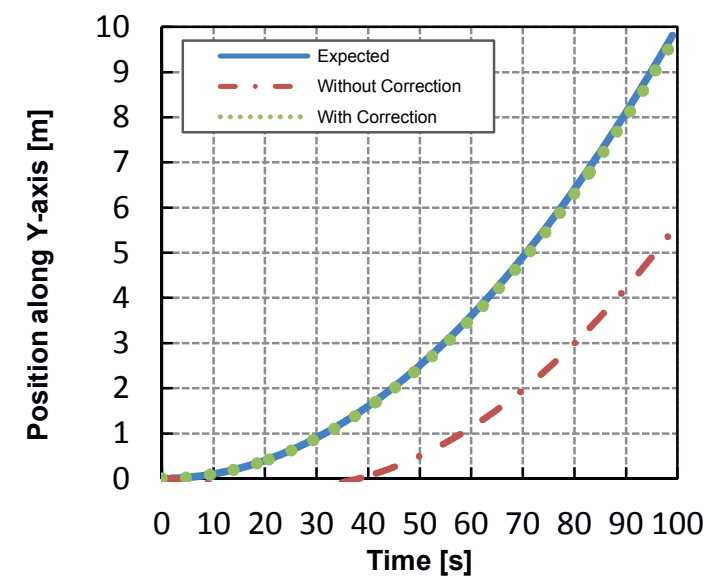

Fig. 2: Comparison with only acceleration and without the effect of any rotation on any of the axis. 
When this is again simulated using EKF, the results shows that, by precise handling of the noise, the EKF can correct or update the data close to the expected nature, as shown in figure 2 with the green line.

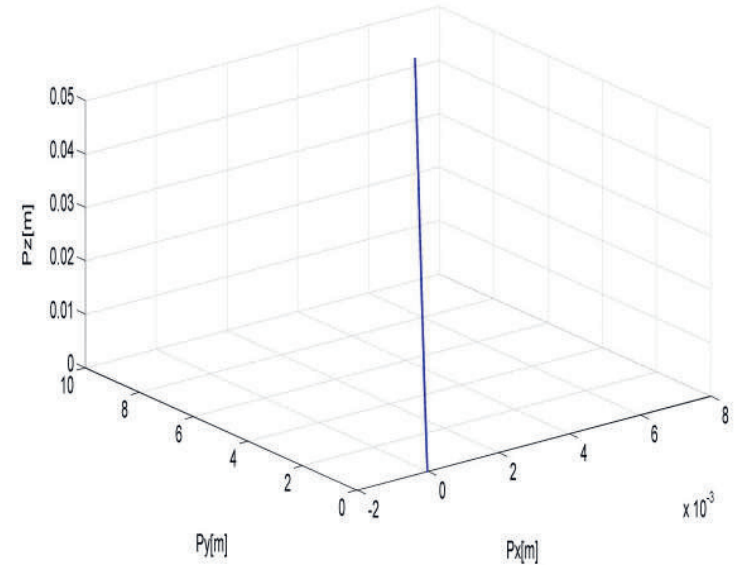

Fig. 3: 3D positioning with acceleration in $y$ axis without any rotation.

Figure 3 shows the three-dimensional positioning of the simulated developed system with constant acceleration in y-axis without any rotation. It can be observed that there is a very small drift and added noise in the other two axis. These drifts and noises can be reduced by precisely controlling the noise parameters. This can be better understood from figures 4 and 5 .

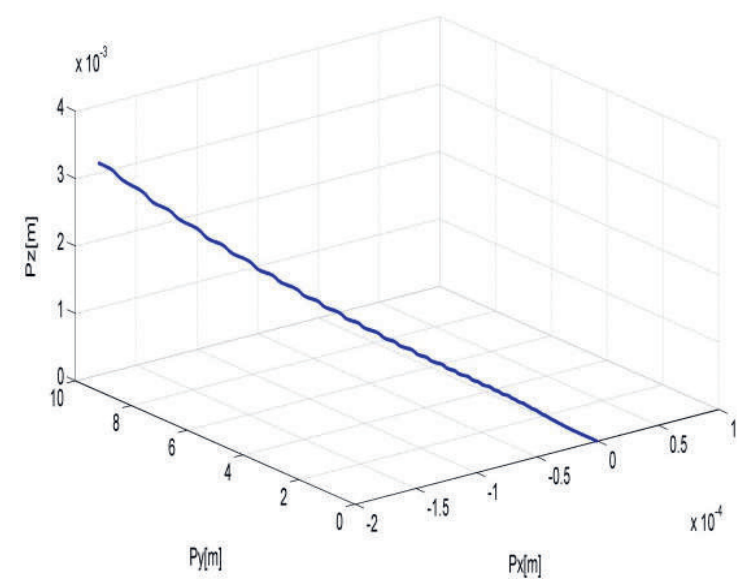

Fig. 4: 3D positioning with acceleration in $y-$ axis with rotation around the same axis.

Figure 4 and 5 show the 3D positioning with acceleration and rotation in y-axis. From figure 4 , it can be seen that due to a less optimized noise parameter the trajectory drifts in the range of $20^{*} 10^{-5}$, whereas, after a small change in the noise parameters, the drift is reduced to the range of $4^{*} 10^{-6}$. By further optimization of the noise parameters, the trajectory can be brought even closer towards the expected trajectory.

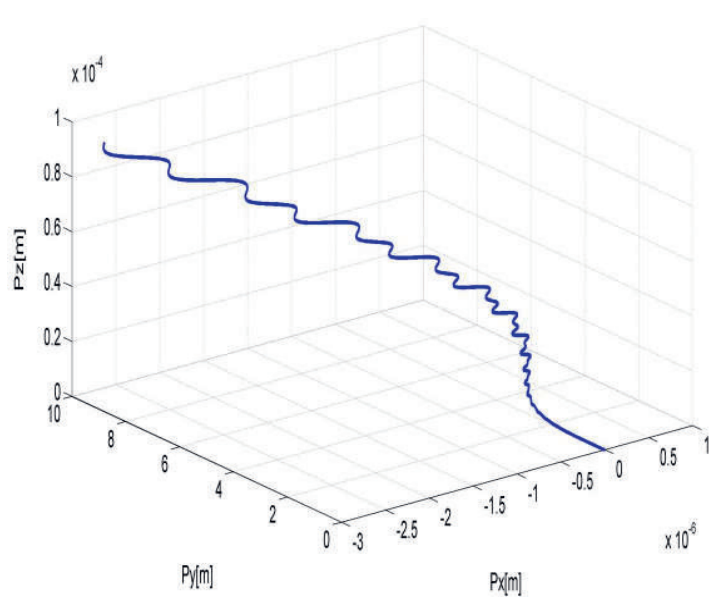

Fig. 5: 3D positioning with acceleration in $y$ axis with rotation in the same axis with better noise model.

\section{Conclusion}

In this work, we used EKF to fuse non-linear data that represent the kinematics of an IMU and combined it with robustness tests to achieve smooth estimates that will be useful in visually challenging and degraded GPS environments. The first simulations showed that by precisely handling or controlling the noise parameters within the EKF algorithm, the drift and the noise in the estimated output can be optimized towards the expected nature. Future work will encompass exploitation of real sensor data in real environments using EKF.

\section{Acknowledgements}

Results of this paper also originate from the research project LEOBEL, which is funded by the German


Federal Environmental Foundation (DBU Deutsche Bundesstiftung Umwelt) under the reference number AZ30799. 


\section{References}

[1] S. F. Reinecke, U. Hampel, 'Instrumented flow following sensor particles with magnetic position detection and buoyancy control', J Sens Sens Syst, 5 (2016), 213220.

[2] H. Nokland, 'Non-linear observer design for GNSS and IMU integration', Norwegian University of Science and Technology, 2011. [3] Md. G. Hasan, et.al, 'Evaluation of a low cost MEMS IMU for indoor positioning system', IJESE, 1(2013)11, 46-50.

[4] H. G. Marina et.al, 'UAV Attitude Estimation Using Unscented Kalman Filter and TRIAD', IEEE Transactions on Industrial Electronics, vol. 59, (11), 2012

[5] A. Chambers et.al, 'Robust Multi-Sensor Fusion for Micro Aerial Vehicle Navigation in GPS-Degraded/Denied Environments', Proceedings of American Control Conference, June 2014.

[6] C.Kang and C. Park, 'Attitude Estimation with Accelerometers and Gyros Using Fuzzy Tuned Kalman Filter', ISBN 978-963-311-3691, Proceedings of the European Control Conference 2009, 3713-3718. 